\title{
DETECÇÃO DE FALHAS ESTRUTURAIS DE UM PRÉDIO DE DOIS ANDARES UTILIZANDO SISTEMA IMUNOLÓGICO ARTIFICIAL
}

\section{DETECTION OF STRUCTURAL FAULTS OF A TWO-STORY BUILDING USING ARTIFICIAL IMMUNE SYSTEM.}

Thiago Carreta Moro ${ }^{1}$, Fábio Roberto Chavarette ${ }^{1}$, Luiz Gustavo Pereira Roéfero ${ }^{1}$, Roberto Outa ${ }^{2}$

${ }^{1}$ Universidade Estadual Paulista - UNESP. ${ }^{2}$ Faculdade de Tecnologia de Araçatuba-FATEC.

E-mail: thiago.moro@unesp.br

RESUMO - Este trabalho apresenta a proposta inovadora para o desenvolvimento de SHMs com foco em ciber-sistemas físicos aplicados em um prédio de dois andares baseado em técnicas de computação inteligente $(\mathrm{Cl})$, o algoritmo de seleção negativa oriundo do Sistema Imunológico Artificial, para realizar a análise e monitoramento da integridade estrutural em um edifício.

Palavras-chave: Detecção de Falhas, Monitoramento de Integridade Estrutural, Sistema Imunológico Artificial

ABSTRACT - This work presents the innovative proposal for the development of SHMs with focus on physical cyber systems applied in a two-story building based on Intelligent Computing (Cl) techniques, the negative selection algorithm from the Artificial Immune System, to perform the analysis and monitoring of structural integrity in a building Keywords: Detection, Structural Health Monitoring, Artificial Immune System 


\section{INTRODUÇÃO}

Com a quarta revolução industrial o modelo ciber-físicos exigiu a reestruturação da indústria do século XXI. As Industrias 4.0, definidas pela implementação do modelo ciber-fisicos, são computacionais e constituídas de sistemas colaborativos nos quais as operações são monitoradas, coordenadas, controladas e integradas por comunicação e núcleos computacionais com o objetivo de transformar a interação do ser humano com o mundo físico. (MAGRUK, 2016)

Assim, sistemas de monitoramento e diagnóstico tendem a se tornar parte do sistema ciber-físico num futuro próximo, como resultado do desenvolvimento de novas tecnologias, processamento de sinais e métodos de interpretação para métodos eficientes de análise da integridade das estruturas, prevenindo catástrofes e / ou acidentes, garantindo assim a vida das pessoas e evitando perdas econômicas. (HUXTABLE, 2016)

Dentro desse conceito que se estabelece o Sistema de Monitoramento de Integridade Estrutural (MIE). De uma forma concisa, o MIE, é descrito como um método de implementação de estratégias, na identificação de falhas estruturais, sendo capaz de executar tarefas como: aquisição e processamento de dados, validação e análise; possibilitando a identificação e interpretação de mudanças adversas em uma estrutura, visando facilitar tomada de decisões e garantir a segurança, em métodos não destrutivos. (GOPALAKRISHNAN, 2011)

Dentro da literatura é verificado muitos trabalhos tradicionais com foco no MIE como estabelecidos em Balageas et al. (2010), e Abreu (2014). Estes trabalhos têm por base: inspeção ultrassônica, raio $X$, acústicos testes de emissões, entre outras técnicas.

Estes trabalhos tradicionais apresentam uma boa metodologia e bons resultados, entretanto estas técnicas apresentam um alto custo com aparelhos específicos e neste caso o pior ponto, não são capazes de abranger a totalidade de grandes estruturas civis, como o caso de uma ponte ou um prédio foco deste trabalho.

Este trabalho apresenta a proposta inovadora para o desenvolvimento de SHMs com foco em ciber-sistemas físicos aplicados em um prédio de dois andares, baseada em técnicas de computação inteligente $(\mathrm{Cl})$, o algoritmo de seleção negativa (FORREST et al., 1994) para realizar a análise e monitoramento da integridade estrutural em um edifício. A partir da aquisição e processamento dos sinais, aplica-se o algoritmo de seleção negativa (ASN), para identificar e caracterizar as falhas através da dinâmica estrutural. Para avaliar este método, utilizou-se a técnica de shear building associado a materiais inteligentes que atuam como sensores.

\section{METODOLOGIA EXPERIMENTAL}

Independentemente do modo, as falhas estruturais são consequência de fatores naturais e forçantes que modificam os parâmetros espaciais e consequentemente ocasionam a repercussão nas características dinâmicas estruturais: funções de respostas em frequência, frequências de ressonância, razão de amortecimento e modos próprios da estrutura. (ATALLA; SGARD, 2010 ) .

Por isso, a metodologia tem por objetivo a captação da reposta dinâmica da estrutura em um estado normal e em um estado de falha, respeitando a avaliação não destrutiva.

\subsection{APARATO EXPERIMENTAL}

Este artigo apresenta a proposta de uma metodologia baseada no modelo de shear building de um prédio de dois andares representado na Figura 1 e seus aparatos experimentais representado pela Figura 2. 
Figura 1. Modelo Experimental
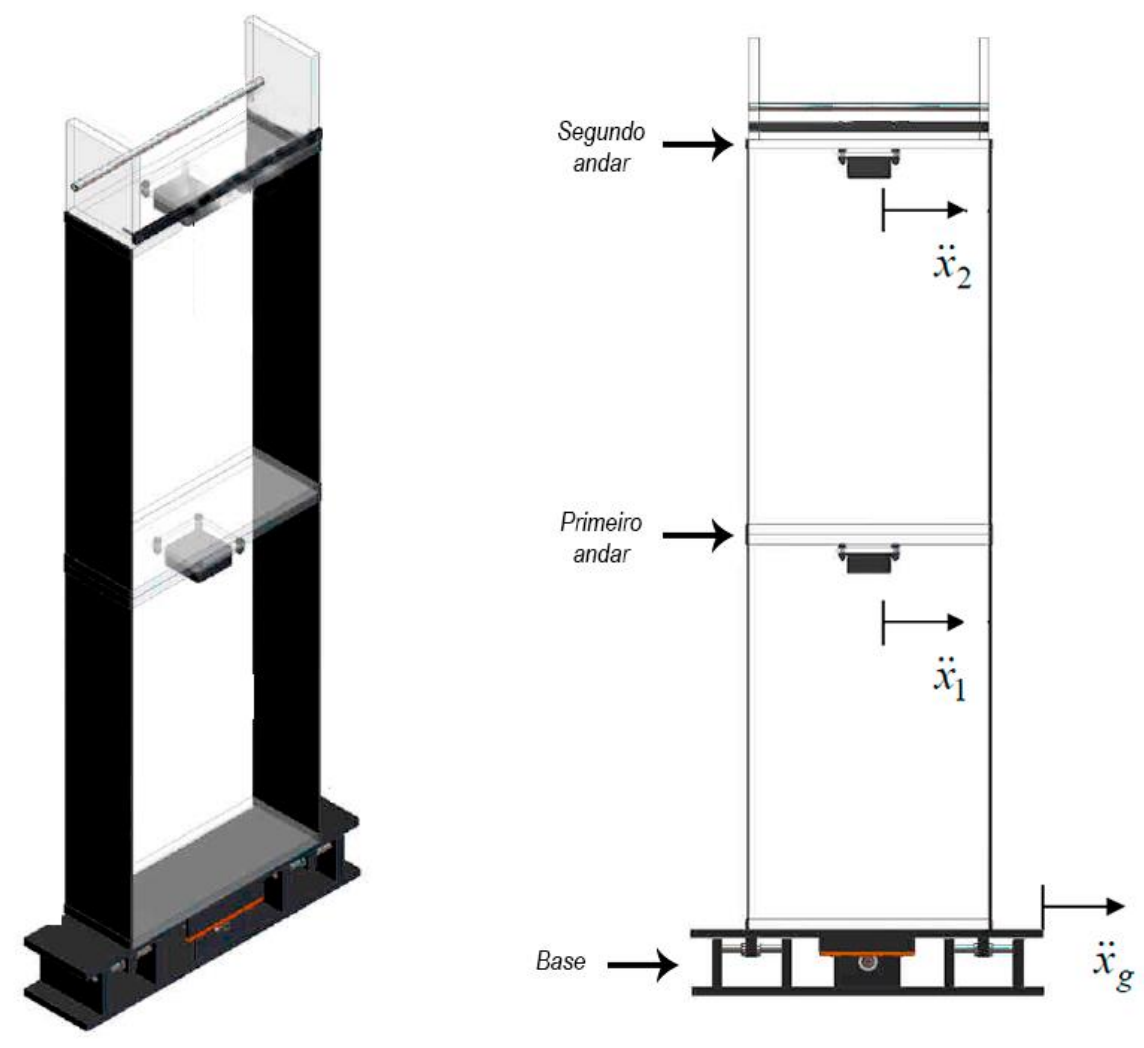

Fonte: elaborado pelo autor

Figura 2. Aparatos Experimentais
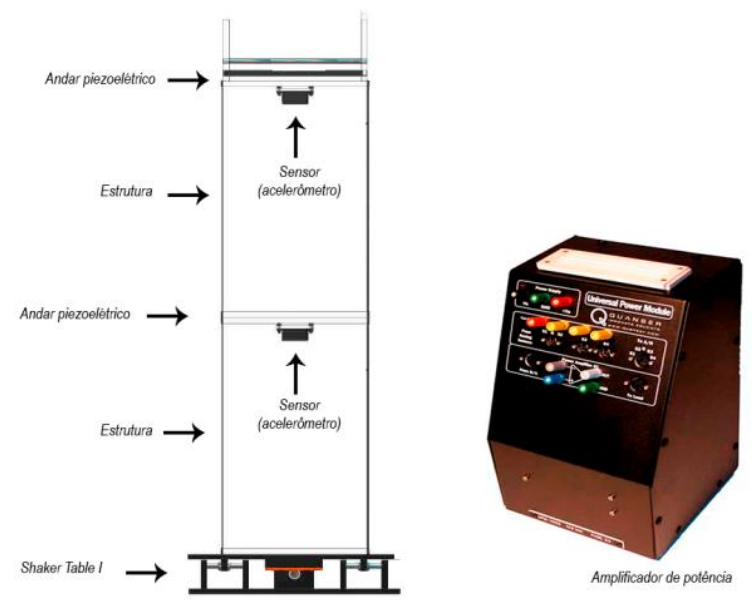

Fonte: elaborado pelo autor

O Modelo de shear building caracteriza uma estrutura real em uma ótica reduzida e com algumas simplificações destacadas por Silvia como:

- Somente o deslocamento horizontal
- As massas são concentradas sobre os nós estruturais

- Despreza-se as massa das colunas,

- Rigidez infinitas das lajes, 
- As ações são aplicadas apenas nos planos dos pórticos,

- A curva de tensão/deformação é considerada elasto- plástica ideal

Através dessas simplificações que se baseia a estrutura utilizada, sendo esta composta por uma estrutura manufaturada pela Quanser Consulting Inc, constituída por uma armação de aço que representa um edifício flexível desenvolvida com dois andares.

A estrutura é dimensionada com $1125 \mathrm{~mm}$ de altura, sendo cada coluna de aço com uma seção de $1,75 \times 108$ milímetros. A massa total da estrutura é de $4,52 \mathrm{~kg}$, onde a massa do primeiro piso $m_{1}$ é de $1,16 \mathrm{~kg}$, a massa do segundo piso $m_{2}$ é $1,38 \mathrm{~kg}$.

O shaker table, ou mesa de agitação, localizado na base do modelo de edifício, produz uma força externa na base da estrutura, definindo uma aceleração $\ddot{x_{g}}$. Este agitador é responsável neste trabalho por excitar a estrutura analisada.

Cada piso dos andares, apesenta um capacitor DC acelerômetro piezoeléctrico. Sendo este material inteligente utilizado para captar as acelerações $\ddot{x_{1}}$ e $\ddot{x_{2}}$ resultantes da excitação da mesa de agitação.

Além dos elementos que compõem o modelo do edifício de dois andares, usou-se um amplificador de potência, responsável por alimentar a mesa de agitação. 0 amplificador, de modelo UPM 1503 manufaturado pela Quanser Consulting In.

O modelo do edifício junto com o amplificador, foi fixado em uma mesa inercial. Esta tem por objetivo garantir a estabilidade do experimento, reduzindo os ruídos experimentais.

\subsection{PROCEDIMENTO EXPERIMENTAL}

Para uma análise estabelecida em variadas amplitudes de excitação, optou-se por uma excitação do tipo El centro, representada pelo modelo estocástico de terremotos de Kanai-Tajimi (Wg), definido, segundo Kanai (1961), por

$$
W g=\frac{\sqrt{S o\left(2 \zeta_{g} \varphi_{g}+\varphi_{g}^{2}\right)}}{s^{2}+2 \zeta_{g} \varphi_{g}+\varphi_{g}^{2}}
$$

Este modelo foi reproduzido pela mesa de excitação e tem por parâmetros iniciais $S_{0}=$ $0.005, \zeta_{g}=0.5$ e $\varphi_{g}=15 \mathrm{rad} / \mathrm{s}$.

Qualquer estrutura é excitada por diferentes frequências, oriundas por diferentes fontes de excitação, como por exemplo a ação do vento, a ação da movimentação de veículos, a solicitação estrutural entre outros.

Por isso a escolha de uma excitação sísmica que neste caso, por apresentar um tempo e uma potência diminuta, não apresenta risco a estruturais ao modelo predial e possibilita um monitoramento completo da estrutura frente às várias fontes de excitação.

Com relação a captação dos sinais, os sinais não próprios da estrutura representam os modos de falhas possíveis que alteram os parâmetros espaciais. Buscando um ensaio não destrutivo, utilizou-se massas adicionais nos andares da estrutura modificando a resposta dinâmica.

Com relação aos sinais próprios, uma estrutura normal, dentro da literatura, pode apresentar um acréscimo diminuto de massa, oriundo do desvio padrão da heterogeneidade dos materiais que a compõem, desde que não haja reflexos na dinâmica estrutural. Por isso, utilizou-se um acréscimo diminuto de massa na captação de sinais normais (cerca de $1 \%$ do peso dos pisos), além da estrutura sem acréscimos de massa.

Portanto, este procedimento experimental tem por possibilidades a captação de 121 sinais. Destes, 4 sinais são da estrutura em estado normal e 117 em estado de falha.

Após a captação dos sinais, aplicou-se a transformada de Fourier. Através da mudança para o espectro de frequência é possível uma análise pico a pico da amplitude de resposta 
É dentro deste contexto tecnológico e inovador que nasce o Algoritmo de Seleção

4. SISTEMA IMUNOLOGICO ARTIFICIAL: ALGORITIMO SELEÇÃO NEGATIVA

O corpo humano é constituído por inúmeros sistemas que, em sincronia, mantem o organismo funcionando sem defeitos fatais. Dentre os Sistemas, o Imunológico é sem dúvida um dos mais importantes a sobrevivência dos seres vivos.

Isto é destacado pelo Calich et al. (2001), sem um mecanismo de defesa os animais estariam a mercê de inúmeros agentes infeciosos presentes no ambiente que invadiriam o corpo humano e causariam o colapso dos sistemas que o compõem.

Esse complexo sistema, chama muito a atenção do ponto de vista biológico e de engenharia devido as propriedades que incluem o reconhecimento de padrões, autonomia, deteç̧ão de anomalias, tolerância a ruído, entre muitas outras (DE CASTRO, 2001).

Assim, com advento da Computação Inteligente, em 1990 surge o Sistema Imunológico Artificial (SIA) que tem por princípio algoritmos inovadores com paráfrases do Sistema Imunológico Biológico, absorvendo assim as propriedades desse importante sistema humano.

Segundo De Castro (2002) o SIA é um marco de evolução da computação inteligente sendo denominado por ele como uma engenharia imunológica.

Isto é baseado na característica de metassíntese, o qual define ferramentas de solução ou otimização de problemas baseando-se nas características do próprio problema, para posterior resolução. Com isso, foge do paradigma da reconstrução parcial ou total do sistema, buscando implementar modelos pragmáticos que preservem algumas das propriedades essenciais e que se mostrem passiveis de implementação computacional e eficaz no desenvolvimento de ferramentas de engenharia.
Negativa (ANS).

Este algoritmo proposto por Forrest et al. (1994), baseia-se no processo de seleção que ocorre no interior do Timo no processo de diferenciação das células linfáticas em próprias e não próprias ao corpo (LIMA, 2016).

Assim o ANS presenta a característica de detectar mudanças em estados de sistemas atuando no reconhecimento de padrões e na detecção de anomalias sendo, além do uso e materiais inteligentes, o diferencial e revolucionário do MIE para atuar na área estrutural.

Estas propriedades são consequências do funcionamento do algoritmo por meio do método de casamento. $O$ critério de casamento ou combinação, segundo Forrest et al. (1994), é utilizado para avaliar afinidade entre as cadeias (antígeno e anticorpo) e comprovar se estas são semelhantes ou iguais. Este casamento pode ser perfeito ou parcial.

Se o casamento for perfeito, significa que as duas cadeias analisadas apresentam os mesmos valores e logo ambas devem ser perfeitamente iguais. Entretanto, se apenas uma quantidade de posições entre os padrões possuírem o mesmo valor este casamento é definido como parcial.

$\mathrm{O}$ antígeno $(\mathrm{Ag})$ é o sinal a ser analisado no algoritmo de seleção negativa e pode ser representado pela expressão (2). Os detectores representam os anticorpos (Ab) e são expressos de acordo com a expressão (3) (de Castro, 2001).

$$
\begin{gathered}
A g=A g_{1}, A g_{2}, A g_{3}, A g_{4}, \ldots, A g_{l} \\
A b=A b_{1}, A b_{2}, A b_{3}, A b_{4}, \ldots, A b_{l}
\end{gathered}
$$

Neste caso, a quantidade de posições iguais obedece a um limiar preestabelecido e denominado taxa de afinidade.

Conforme Lima (2017), o casamento perfeito entre as cadeias e dados é quase impossível de ser alcançado experimentalmente, devido as inúmeras variáveis que permeiam e alteram os dados empíricos. Por isso a utilização do casamento 
parcial e a taxa de afinidade mostram-se muito mais vantajosos.

A taxa afinidade utilizada como o grau de semelhança necessário para que ocorra o casamento parcial, avaliada para os números reais, é definida, de acordo com Lima (2014) como:

$$
T A f=\left(\frac{A n}{A t}\right) * 100
$$

Sendo:

$T A f$ : taxa de afinidade;

An : número de cadeias normais no problema (cadeias próprias);

At : número total de cadeias no problema (cadeias próprias e não-próprias)

Além da Taxa de afinidade, para analisar os padrões de cadeia é utilizado a Afinidade total, definida como:

$$
A f_{T}=\frac{\sum_{i=1}^{L} V c}{L} * 100
$$

Sendo:

$A f_{T}: \%$ de afinidade entre os padrões analisados;

L : quantidade total de variáveis;
Vc : variáveis casadas;

$\sum_{i=1}^{L} V c$ : somatória (quantidade) de variáveis casadas.

Assim, caso $A f_{T}$ for maior ou igual ao TAf ocorre o casamento perfeito e as cadeias são consideradas semelhantes. Por outro lado, se a $A f_{T}$ for menor que a $T A f$, o detector não reconhece a cadeia, e logo não ocorre o casamento entre os padrões.

Utilizando, portanto, o casamento parcial, o ANS é segmento em duas fases iterativas: Censoriamento e Monitoramento

$\mathrm{Na}$ fase de Censoriamento do ASN, representada pela Figura 1, são definidos os detectores próprios, conhecidos também como cadeias próprias (S), representam a condição normal do problema. Em seguida, é produzido o conjunto de detectores $(R)$, que possuem a capacidade de reconhecer padrões não próprios. Portanto, com a leitura dos dados, verifica-se a afinidade de cadeias aleatórias, comparando estas cadeias ao conjunto de cadeias próprias.

Se a afinidade estiver a cima de um limiar preestabelecido a cadeia é rejeitada. Entretanto se a afinidade estiver abaixo do limiar, a cadeia é adicionada ao conjunto de detectores, denominado também por Baseline, sendo utilizada na classificação durante o monitoramento dos dados.

Figura 3. Fluxograma Censoriamento

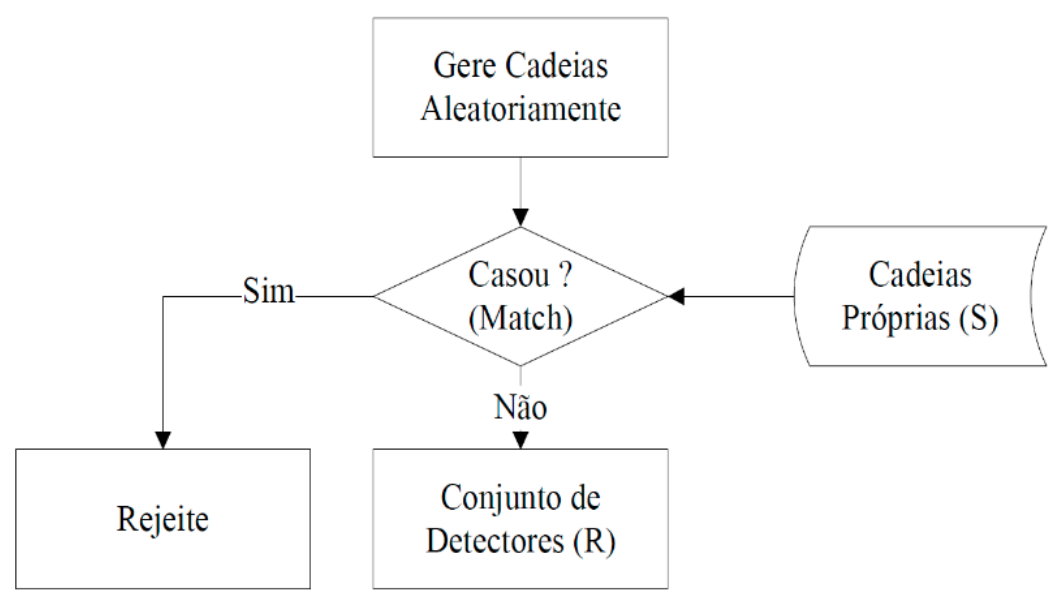

Fonte: Lima (2016) 
$\mathrm{Na}$ fase de monitoramento, representada pela Figura 2 , é realizado o monitoramento dos dados com o objetivo de identificar anomalias de comportamento das amostras. Assim, com o conjunto de detectores, oriundos da fase de Censoriamento, classificar esta anomalia.
Deste modo, comparando as cadeias protegidas (S) com o conjunto de detectores $(\mathrm{R})$, afere-se a afinidade entre as cadeias. Caso a afinidade seja superior a um limiar preestabelecido, e identificado e classificado um elemento não próprio.

Figura 4. Fluxograma Monitoramento

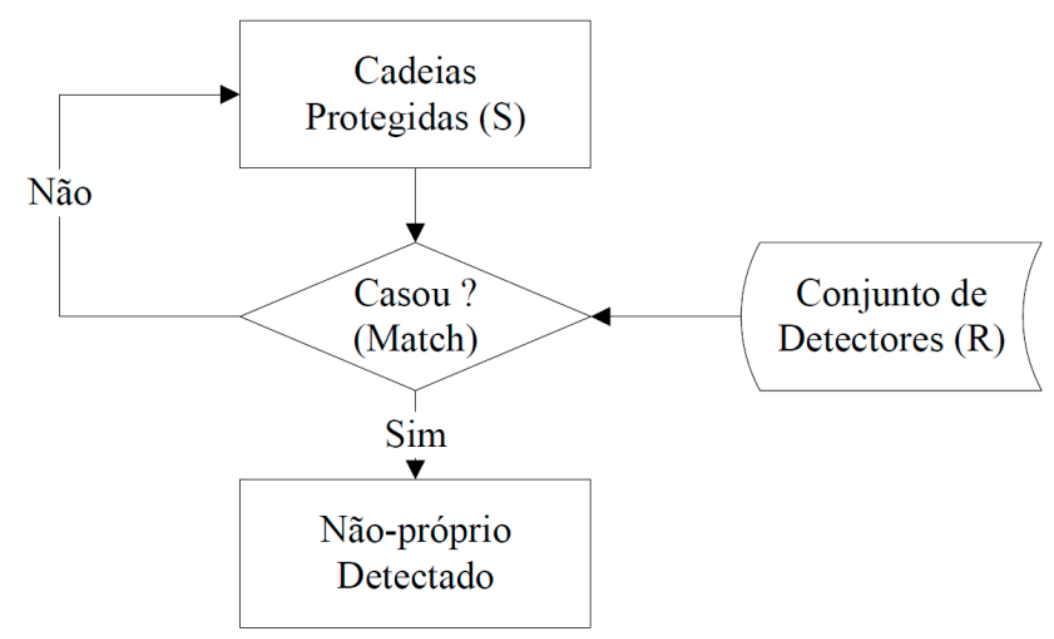

Fonte: Lima (2016)

\section{RESULTADOS E DISCUSSÃO}

A evolução do MIE neste trabalho tem por vertente a utilização dos materiais inteligentes para a captação de dados e em segundo ponto a utilização do Sistema Imunológico Artificial para um processamento mais eficiente.

\subsection{MATERIAIS INTELIGENTES}

Logo por resultados iniciais, a captação de dados mostrou-se eficaz e economicamente viável, pois possibilitou o sensoriamento global da estrutura com uso de dois sensores piezoeléctricos.
Isso é comprovado pela Figura 5, ao plotar um sinal próprio (sinal da estrutura normal) da estrutura e um sinal não próprio( sina da estrutura em modo de falha) da estrutura, após a Transformada de Fourier, verifica-se uma diferença de amplitude entre os sinais indo ao encontro de Atalla e Sgard (2015): a mudança do parâmetro espacial (acréscimo de massa) altera a resposta em frequência; dando confiabilidade ao sistema de captação baseado em materiais inteligentes. 
Figura 5. Sinais captados da estrutura

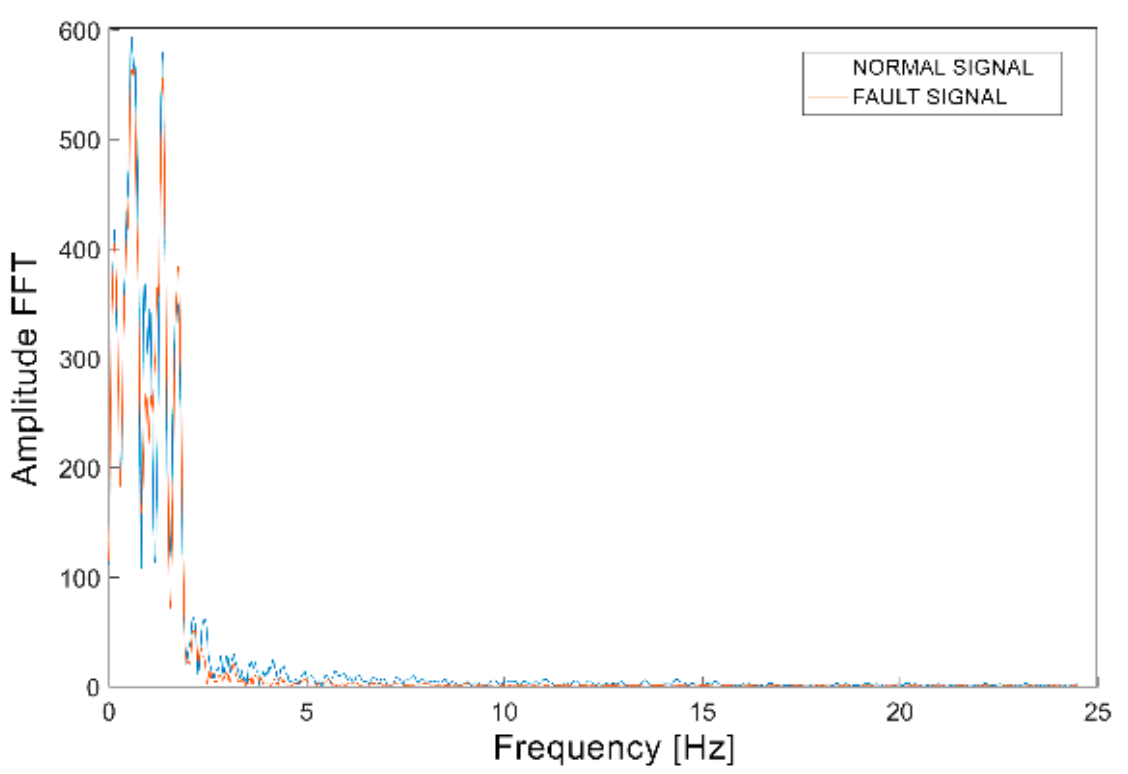

Fonte: Os autores.

Relativamente em uma estrutura predial seria necessário o uso de mais sensores piezoeléctricos, entretanto, seu domínio global e possibilidade de monitoramento instantâneo já supera o uso das técnicas tradicionais que apresentam deficiência de domínio e alto índice econômico.

\subsection{ALGORITMO DE SELEÇÃO NEGATIVA}

Para verificar a eficiência do algoritmo de seleção negativa, recorreu-se a subdivisão do processamento dos sinais estruturais em três situações: Processamento Sequenciado, Randômico Não Próprio e o Randômico Decrescente Próprio.

Para garantir a normalidade com base na curva padrão dos resultados amostrais, baseado em SNEDECOR et al. (1989), realizou-se trinta iterações do algoritmo com os dados estruturais (sinais) para cada tipo de processamento.

\subsubsection{PROCESSAMENTO SEQUENCIADO}

O Processo Sequenciado tem por princípio uma verificação do funcionamento correto do algoritmo. Este processamento é caracterizado pela entrada sequencial de sinais, ou seja, os sinais que alimentam o algoritmo são ordenados do melhor caso para o pior caso de falha.

O processo sequenciado foi definido com 4 sinais próprios e 117 sinais não próprios.

Assim obteve-se por resultado o gráfico de porcentagem de acerto, representado na Figura 6. Observando o valor médio representado pela linha vermelhas, houve uma Taxa de acerto de $100 \%$ com um tempo de processamento de 0.05 segundos. 
Figura 6.

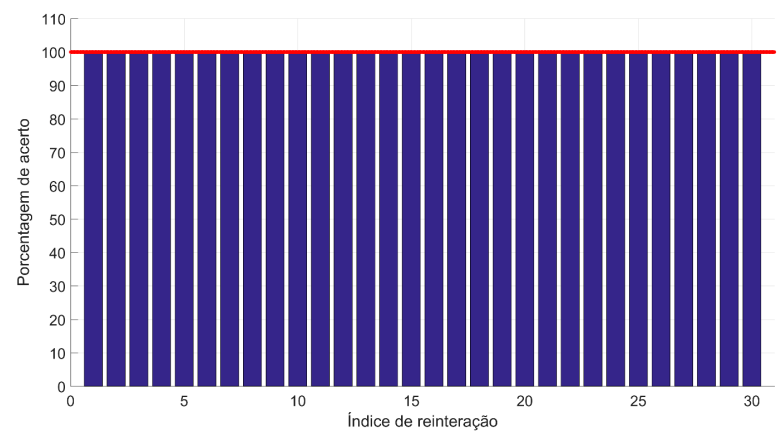

Fonte: Os autores.

Observando 0 processamento sequenciado, o algoritmo apresentou uma eficiência máxima, validando, portanto, o funcionamento correto do algoritmo. Ao alimentar o ANS com os mesmos sinais estabelecidos na memória inata do algoritmo (Fase de Censoriamento), deve-se haver o auto reconhecimento e consequentemente o acerto máximo (FORREST et al., 1994).

\subsubsection{PROCESSAMENTO NÃO PRÓPRIO}

Neste método foi utilizado fixamente quatro sinais normais e variado, randomicamente, o número de sinais não próprios estruturais a cada iteração. Com esse método, é avaliado a eficiência do algoritmo para variadas taxas de afinidade e, também, a relação entre o processamento e o conhecimento a respeito dos sinais com falhas.

Por isso a Tabela 1, representa para as trintas interações realizadas, o número de sinais com falha usados.

Tabela 1. Numero de sinais com seus índices

\begin{tabular}{|c|c|c|c|c|c|c|c|c|c|c|c|c|c|c|c}
\hline Iteração & 1 & 2 & 3 & 4 & 5 & 6 & 7 & 8 & 9 & 10 & 11 & 12 & 13 & 14 & 15 \\
$\begin{array}{c}\text { Sinais } \\
\text { Não proprios }\end{array}$ & 7 & 105 & 76 & 16 & 113 & 73 & 23 & 81 & 35 & 66 & 14 & 105 & 10 & 25 & 25 \\
\hline & & & & & & & & & & & & & & \\
\hline Iteração & 16 & 17 & 18 & 19 & 20 & 21 & 22 & 23 & 24 & 25 & 26 & 27 & 28 & 29 & 30 \\
\hline $\begin{array}{c}\text { Sinais } \\
\text { Não proprios }\end{array}$ & 33 & 107 & 116 & 21 & 85 & 66 & 50 & 41 & 15 & 99 & 37 & 71 & 117 & 97 & 92 \\
\hline
\end{tabular}

Obteve-se por resultado o gráfico de porcentagem de acerto, representado na Figura 10.

Observando o valor médio houve uma Taxa de acerto de $100 \%$ com um tempo de processamento de 0.0504 segundos.

Figura 7. 


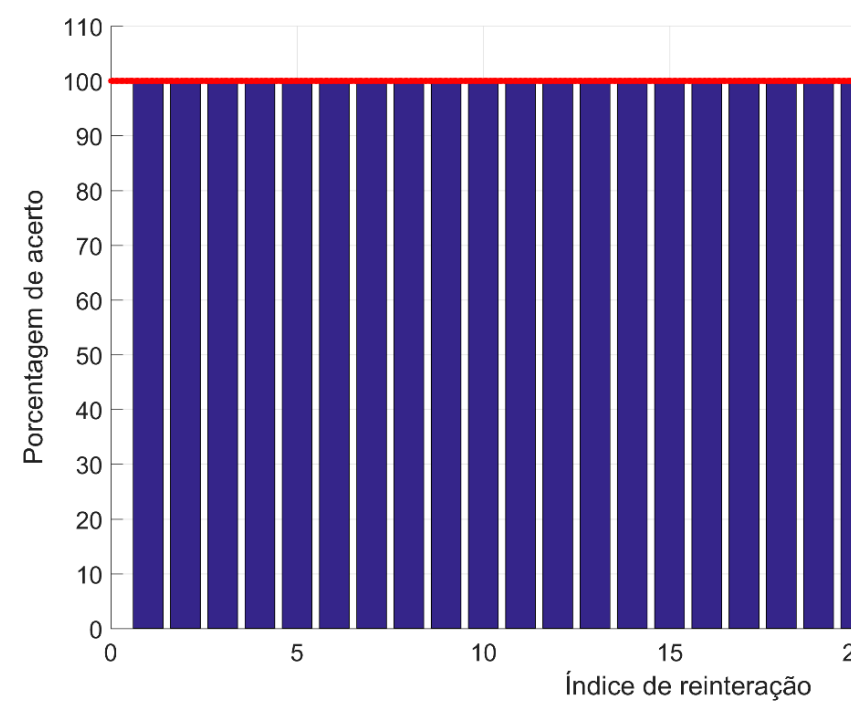

Fonte: Os autores.

O processamento Randômico não próprio, caracteriza uma analise a respeito da precisão do algoritmo em várias taxas de afinidade (diversificados valores de sinais com falha). Observando o gráfico de acertos, denota-se que a variação do número de sinais não próprios, que é avaliada, não influencia a taxa de acerto, afinal esta foi de $100 \%$.

Logo, independentemente do modo de falha que a estrutura ficar susceptível o MEI proposto o detectará.

\subsubsection{PROCESSAMENTO PRÓPRIO}

Neste método foi utilizado fixamente 117 sinais com falhas e variado, decrescentemente, o número de sinais próprios estruturais a cada interação. Com esse método, é avaliado a eficiência do algoritmo para uma situação em que o mesmo não apresenta um aprendizado completo a respeito da estrutura monitorada.

Como este banco é constituído por quatro sinais normais desenvolveu-se uma entrada decrescente do número de sinais sem falha iniciando com quatro sinais, e finalizando com um sinal, o índice de acerto médio é representado concisamente na Figura 8 para cada número de sinais normais.

Figura 8. Comparação do próprio

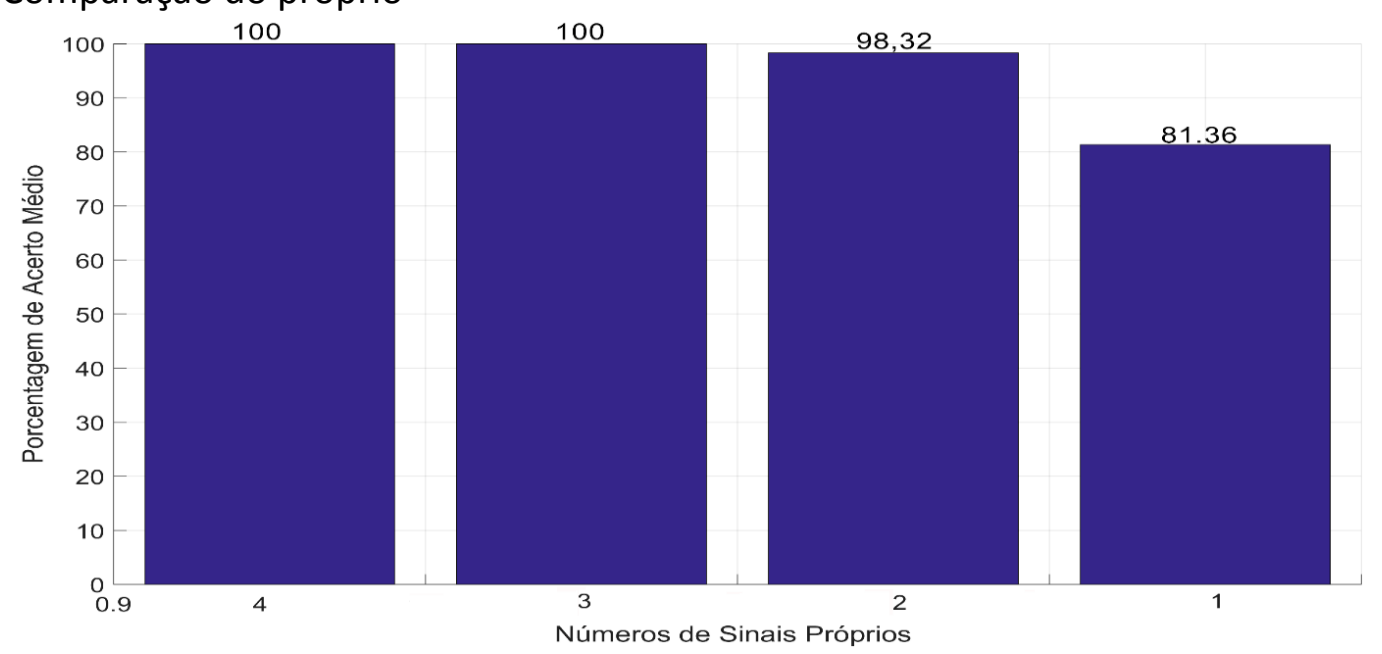

Fonte: Oa autores.

Através desses gráficos, denota-se que a precisão do Algoritmo de Seleção Negativa é oriunda do aprendizado desenvolvido na etapa de Censoriamento. Logo, é evidente que quanto mais sinais próprios, maior será aprendizado e, consequentemente, o algoritmo terá uma maior precisão e acurácia.

\section{CONSIDERAÇÕES FINAIS}

Este trabalho apresenta uma inovadora constituição de Monitoramento de Integridade Estrutural alicerçado em sensores de materiais inteligentes e na implementação do Sistema Imunológico Artificial indo ao encontro da filosofia ciber-física da quarta revolução industrial. 
Isto é demonstrado pelos resultados obtidos afinal mesmo tendo um baixo aprendizado por parte do ANS, O MEI proposto apresentou uma acurácia e precisão superior a $80 \%$ associado a um tempo de processamento inferior a um segundo.

Portanto, observando o trabalho total, este Sistema Monitoramento da Saúde Estrutural aplicado ao modelo predial, caracteriza uma revolução ao ramo estrutural, pois, transforma o principal o determinante estrutural em um "corpo humano". Ou seja, em uma máquina complexa e perfeita com todas as suas partes funcionando em sincronia e sem falhas potenciais.

\section{AGRADECIMENTO}

Este trabalho não seria possível sem o apoio financeiro oferecido pela Fundação de Amparo à Pesquisa (FAPESP), através dos processos 2018/06573-6 e 2019/10515-4. Por isso, os autores agradecem o apoio e a confiança depositada nesta pesquisa.

Além disso, agradeço a UNESPUniversidade Estatual Paulista por proporcionar a oportunidade de estar no meio acadêmico e suporte técnico para esta pesquisa.

E por último, mas não menos importante agradeço ao Sisplexo, o laboratório onde trabalho e onde tenho uma segunda família.

\section{REFERÊNCIAS}

ABREU, C. C. E., CHAVARETT, F. R., ALVARADO, F. V., DUARTE, M. A. Q., LIMA, F. P. A. , Dual-Tree Complex Wavelet Transform Applied to Fault Monitoring and Identification in Aeronautical Structures. International Journal of Pure and Applied Mathematics, v. 97, n.1, p. 89-97, 2014. https://doi.org/10.12732/ijpam.v97i1.9

ATALLA, N.; SGARD, F. Finite element and boundary methods. In:

BALAGEAS, D.; FRITZEN, C.-P.; ;GUEMES, A.. Structural health monitoring, [S.I.]: John Wiley Sons, 2010, v. 90.
CALICH, V. L. G.; VAZ, C. A. C.; ABRAHAMSOHN, I. D. A.; BARBUTO, J. A. M.; ISAAC, L.; RIZZO, L. V.; MACEDO, M. S.; RUSSO, M.; JANCAR, S.Imunologia. São Paulo: Revinte, 2001.

CASTRO, L. N.Engenharia imunológica: desenvolvimento e aplicação de ferramentas computacionais inspiradas em sistemas imunológicos artificiais. 2001. Tese (Doutorado) - Universidade Estadual de Campinas, Campinas, 2001.

DE CASTRO, L. N.; TIMMIS, J. Artificial immune systems: a new computational intelligence approach. New York: Springer, 2002. p. 357.

FORREST, S.; PERELSON, A. S.; ALLEN, L.; HERUKURI, R. Self-nonself discrimination in a computer. In Research in Security and Privacy, 1994. Proceedings., 1994 IEEE Computer. https://doi.org/10.1109/RISP.1994.296580

Gopalakrishnan, S., Ruzzene, M., Hanagudh, S., 2011, Computational techniques for Structural Health Monitoring, Springer Science Business Media. https://doi.org/10.1007/978-0-85729-284-1

Huxtable, J., Schaefer, D., 2016. On Servitization of the Manufacturing Industry in the UK. Procedia CIRP 52, 46-51. DOI: 10.1016/j.procir.2016.07.042

https://doi.org/10.1016/j.procir.2016.07.042

Kanai, K., 1961, An empirical formula for the spectrum of strong earthquake motions, Bulletin earthquakes research institute, University of Tokyo 39, 85-95

LIMA, F. P. A.; CHAVARETTE, F. R.; SOUZA, S. S. F.; LOPES, M. L. M.. Monitoring and Fault Identification in Aeronautical Structures Using an Wavelet-Artificial Immune System Algorithm. Probabilistic Prognostics and Health Management of Energy Systems. São Paulo: Springer International Publishing AG, 2017. Chaim, 1-17. 
https://doi.org/10.1007/978-3-319-55852-

$\underline{313}$

MAGRUK, A.Uncertainty in the Sphere of the Industry 4.0-Potential Areas to Research. Business, Management and Education, v. 14, n. 2, p. 275-291, 2016. https://doi.org/10.3846/bme.2016.332

SNEDECOR, G. W.; COCHRAM, W. G. Statistical Methods. $8^{\text {rd }}$ ed. lowa: lowa State

University Press, 1989.

SOCIETY SYMPOSIUM.. leee.Structural

acoustics and vibration. CRC Press, 2015 p.
202-212.

https://doi.org/10.1109/RISP.1994.296580 